\title{
A decrease in care was associated with suicide in mental illness
}

\author{
Appleby L, Dennehy JA, Thomas CS, et al. Aftercare and clinical characteristics of people with mental illness who commit suicide: a \\ case-control study. Lancet 1999 Apr 24;353:1397-1400.
}

\section{Question}

In people with severe mental illness who commit suicide, what is the relation of suicide to aspects of mental health care, clinical features, and historical variables?

\section{Design}

Case control study.

\section{Setting}

Greater Manchester area, UK.

\section{Participants}

Cases were 149 people (median age 38 y) who committed suicide in Greater Manchester within 5 years of discharge from inpatient psychiatric care. Controls were 149 discharged psychiatric inpatients matched to cases by age (within 5 y), sex, clinical diagnosis, and date of admission (within $6 \mathrm{mo}$ ).

\section{Assessment of risk factors}

Data on historical variables, clinical features since final inpatient admission, and details about care after inpatient discharge (aftercare) were obtained from clinical records. Care variables included components of care at the time of suicide, decreases in care (decrease in frequency of follow up and drug dosage, transfer to a setting with less observation, and discharge from follow up), and corresponding increases in care. Predictable risk (risk of suicide perceived by a clinician at the last contact before suicide) was rated independently by 2 psychiatrists blinded to case or control status.

\section{Main outcome measure}

Risk of suicide.

\section{Main results}

The historical and clinical variables that were most strongly associated with suicide were index admission as the first illness, history of self harm, and suicidal thoughts during aftercare. These risk factors and depression in the 3 months before suicide were also associated with each other, therefore adjustment was made (table). Predictable risk rated as moderate or high was not associated with suicide after adjusting for these 4 risk factors. Patients who committed suicide were more likely to have had a decrease in care at their final appointment (table).

\section{Conclusion}

In people with severe mental illness who commit suicide, certain clinical features and historical variables were associated with suicide, including a decrease in care at their final appointment before death.

Risk factors associated with suicide in mental illness

\begin{tabular}{ll}
\hline Risk factors & Odds ratio $(95 \%$ CI)* \\
\hline Index admission as first illness & $2.0(1.1$ to 3.6$)$ \\
History of self harm & $3.1(1.7$ to 5.7$)$ \\
Suicidal thoughts during aftercare & $1.9(1.0$ to 3.5$)$ \\
Decrease in care & $3.7(1.8$ to 7.7$) \dagger$ \\
\hline
\end{tabular}

*Adjustment for history of self harm, suicidal thoughts during aftercare, index admission as first illness, and depression in 3 months before suicide. $\dagger$ Additional adjustment for predictable risk of suicide and hospital of index admission.

Source of funding: North West NHS Trust Executive.

For correspondence:Professor L Appleby, School of Psychiatry and Behavioural Sciences, University Hospital of South Manchester, West Didsbury, Manchester M20 8LR, UK. Fax +44 (0)161 4459263.

Modified version of the abstract also published in Evidence-Based Nursing.

\section{Commentary}

Despite the promulgation of national and international guidelines for suicide prevention, ${ }^{1}$ little evidence exists to show that suicide prevention actually works. Thus, the study by Appleby et al addresses an issue of considerable importance. Given the retrospective design, however, the evidence provided for the importance of prolonging psychiatric aftercare to reduce suicides among patients admitted to hospital with serious mental illnesses is suggestive, not definitive. Furthermore, these results cannot be generalised to developing countries or other settings where the causes of suicide, social support networks, or mental health delivery systems are different from those in Manchester.

The association between suicide and a reduction in the frequency of appointments or the transfer to a less supervised location may be related to the resulting contraction of the patient's social support network or to the patient's feeling of abandonment, not to changes in specific clinical activities such as medication or psychotherapy. The authors could test this hypothesis by determining if people with limited social support (eg, those who are unemployed or living alone) are more likely to commit suicide after reductions in the intensity of care than people with relatively good social support.

Clinicians providing aftercare to discharged psychiatric patients must give additional attention to those at high risk of suicide: patients who have made previous attempts, have only been admitted to hospital once, and currently have depression or suicidal ideation. The finding that $25 \%(37 / 149)$ of patients who committed suicide had services reduced because the treating clinician considered them "well" at the time of their last service contact indicates that we are still unable to judge the appropriate frequency, intensity, and duration of psychiatric aftercare (or provision of other types of social support services) for people we consider at high risk of suicide. The authors' recommendation to prolong aftercare for high risk patients beyond the point of "perceived recovery" is appropriate, but clinicians still have no way of knowing how long is long enough.

Michael R Phillips, MD, MA, MPH Beijing Hui Long Guan Hospital Beijing, People's Republic of China 1 United Nations. Prevention of suicide: guidelines for the formulation and implementation of nationa
strategies. New York: United Nations, 1996. 